\title{
Effect of dietary inulin in the gut microbiota of whiteleg shrimp Penaeus vannamei
}

\author{
Ruth Escamilla-Montes $^{1}(\mathbb{D})$, Aarón Barraza ${ }^{2}(\mathbb{D})$, Antonio Luna-González ${ }^{1}$ (D) Carlos Angulo $^{2}$ \\ Jesús Arturo Fierro-Coronado ${ }^{1}\left(\mathbb{D}\right.$, Genaro Diarte-Plata ${ }^{1} \mathbb{D}$ \& Ma. del Carmen Flores-Miranda ${ }^{3}$ \\ ${ }^{1}$ Instituto Politécnico Nacional, Centro Interdiciplinario de Investigación para el Desarrollo Integral Regional \\ Guasave, Sinaloa, México \\ ${ }^{2}$ CONACYT, Immunology \& Vaccunology group-Centro de Investigaciones Biológicas del Noroeste S.C. \\ Instituto Politécnico Nacional, La Paz, B.C.S., México \\ ${ }^{3}$ Departamento de Estudios para el Desarrollo Sustentable de Zonas Costeras \\ Centro Universitario de la Costa Sur, Universidad de Guadalajara, San Patricio Melaque, Jalisco, México \\ Corresponding author: Antonio Luna-González (aluna@ipn.mx)
}

\begin{abstract}
The effect of dietary inulin on the intestinal bacterial communities of Penaeus vannamei by $16 \mathrm{~S}$ metagenomic analysis was assessed. PCR amplified the V3 region of the bacterial $16 \mathrm{~S}$ rDNA. Sequencing reads were generated using the $2 \times 150$ (300 cycles) for the base-read length chemistry of the Illumina MiniSeq platform. The software Shaman and MicrobiomeAnalyst were used to analyze the sequences. The phylum Proteobacteria and the genus Vibrio were among the most abundant taxonomic ranks for control and inulin treatment. The relative abundance of the phylum Bacteroidetes and genus Ruegeria was lower in inulin treatment concerning the control condition. Alpha and beta indices did not show significant differences between inulin treatment and control conditions. For all samples, most of the bacterial organisms showed the presence of carbohydrate and amino acid metabolism-related genes, and to a lesser extent, of energy, lipid, and cofactors and vitamin metabolism-related genes. The principal metabolic functions were glycine, serine, threonine, glyoxylate and dicarboxylate, purine, pyrimidine, pyruvate, and quorum sensing. The interaction network analysis showed fewer interactions in the inulin treatment concerning control condition. Proteobacteria, Bacteroidetes, Vibrio, and Ruegeria predominated in all samples, and inulin did not change the net microbial diversity in the intestine of $P$. vannamei. Streptomyces, Roseobacter, and Ruegeria showed negative interactions with Vibrio, suggesting their use as probiotics. This study sheds light on the inulin supplement on the essential role of microbiota in the shrimp.
\end{abstract}

Keywords: Penaeus vannamei; metagenome; microbial network; prebiotic; shrimp gut; aquaculture

\section{INTRODUCTION}

In the last years, the whiteleg shrimp Penaeus vannamei culture has been growing steadily throughout the world; however, production has been affected by diseases, mainly viral and bacterial. Antibiotics are a problem in aquaculture due to the emergence of resistant bacterial strains (Aich et al. 2018), so the use of natural and environmentally friendly products, such as prebiotics, is strongly encouraged. Prebiotics are carbohydrates digestible and metabolized only by health-promoting bacteria in the host's gastrointestinal tract, which leads to improvements in growth and gut beneficial bacteria (Gibson 2004, Makki et al. 2018). This fact is due to the digestive enzymes (glucosidases) of the host are not capable of breaking the glycosidic bonds of the monomers of certain prebiotics since the $\mathrm{C} 1$ or $\mathrm{C} 2$ atom of the monomer has a typical configuration (Roberfroid \& Slavin 2000). Therefore, various prebiotics has been used as food additives in aquaculture, such as transgalacto-oligosaccharides (TOS), fructooligosaccharides (FOS), short-chain fructooligo-

Corresponding editor: Jesús Ponce-Palafox 
saccharides (scFOS), xylooligosaccharides (XOS), arabinoxylo-oligosaccharides (AXOS), isomaltooligosaccharides (IMO), mannanoligo-saccharides (MOS), lactose, and inulin (Teitelbaum \& Walker 2002, Vulevic et al. 2004, Ring ø et al. 2010, Abdulrahman \& Ahmed 2015, Azimirad et al. 2016).

Inulin is a natural prebiotic that can modulate gut microbiota, which is very important to maintain shrimp health and fight against the pathogen (Li et al. 2018). In this regard, our research group demonstrated that dietary inulin in shrimp increases the phenoloxidase activity and decreases the prevalence of white spot syndrome virus (WSSV) (Luna-González et al. 2012). Curiously, Indian white shrimp (Fenneropenaeus indicus) post-larvae fed inulin enriched Artemia increased lactic acid bacteria counts in the gut (Hoseinifar et al. 2015). In light of those previous findings, the study of the intestinal microbiota through $16 \mathrm{~S}$ metagenomic analysis is useful to elucidate microbial abundance, diversity, and functional profile in shrimp-fed inulin.

This work aimed to evaluate the effect of dietary inulin on the gut bacterial communities of $P$. vannamei.

\section{MATERIALS AND METHODS}

\section{Experimental shrimp}

Healthy shrimp (without WSSV or Vibrio parahaemolyticus) were collected from the Cuate Machado Aquaculture Farm (Guasave, Sinaloa, Mexico) and transported to the CIIDIR-IPN Sinaloa Aquaculture Laboratory. Animals were acclimatized (10 days) in seawater at 30 of salinity, kept at room temperature and constant aeration, and fed with Camaronin Purina ${ }^{\circledR}$ (30\% protein) at 08:00, 13:00 and 17:00 $\mathrm{h}$.

\section{Inulin inclusion in feed}

The commercial feed (Camaronin, Purina ${ }^{\circledR}$, Mexico, $30 \%$ protein) was pulverized in a coffee grinder to add inulin (2.5 g kg-1 feed). Camaronin had no additives like probiotics or prebiotics. Agave tequilana inulin was obtained from IDEAL, S.A. de C.V., Guadalajara, Jalisco, Mexico. Ingredients were mixed by adding water and gelatin $(40 \mathrm{~g}$ of gelatin and $410 \mathrm{~mL}$ of distilled water $\mathrm{kg}^{-1}$ ), and the pellets were prepared in a meat grinder. The pellets were dried in the laboratory at room temperature with a fan for $24 \mathrm{~h}$. Enough feed for 20 days was prepared and stored at $-20^{\circ} \mathrm{C}$. For the control treatment, inulin was replaced by cellulose $(2.5$ $\mathrm{g} \mathrm{kg}^{-1}$ feed).

\section{Shrimp culture in the laboratory and sample collection}

The bioassay lasted 20 days. Experimental shrimp weighed $7.6 \pm 0.04 \mathrm{~g}$. Sixty liters of filtered seawater $(20 \mu \mathrm{m})$ were added to plastic tanks $(130 \mathrm{~L})$, and constant aeration was maintained. Shrimp were fed twice a day (08:00 and 16:00 h) with Camaronin Purina ${ }^{\circledR}$ (30\% protein), adjusting the amount of feed according to the biomass of the shrimp (5\%). Ten shrimps were placed per tank. The bioassay consisted of two treatments: I) control diet without inulin and II) diet with inulin. Physical and chemical parameters: dissolved oxygen $\left(5.1 \pm 0.4\right.$ to $\left.5.7 \pm 0.45 \mathrm{mg} \mathrm{L}^{-1}\right)$, $\mathrm{pH}$ ( $7.8 \pm 0.5$ to $8.1 \pm 0.3)$, and salinity $(30.38 \pm 1.6$ to $31.15 \pm 1.1)$ were determined daily. The tanks were siphoned every three days, and $50 \%$ of the water was replaced. Survival was determined daily. On day 20, three shrimps per tank were taken (nine individual samples per treatment) for nucleic acid extraction and metagenomic analysis. Each shrimp intestine was removed and placed in a $1.5 \mathrm{~mL}$ tube with $1 \mathrm{~mL}$ of $96 \%$ $(\mathrm{v} / \mathrm{v})$ ethanol and stored at $-70^{\circ} \mathrm{C}$. This study complied with the Mexican Official Standard NOM-062-ZOO1999 for the care and use of laboratory animals.

\section{Nucleic acid isolation}

The shrimp intestine samples were sent to the Research Center for Food and Development (CIAD, Mazatlán, Sinaloa, Mexico), where total DNA was extracted with the CTAB method used for next-generation sequencing (NGS).

\section{Library preparation and sequencing of bacterial DNA}

The $\mathrm{V} 3$ region of the bacterial 16S rDNA was amplified by PCR ( 35 cycles) using the primers V3-338f and V3533r with Illumina adapters and sample-specific tags; indices were also added following the manufacturer's recommendations (Illumina, San Diego, CA, USA). The V3 amplicons were quantified using a Qubit (Thermo Fisher Scientific, Waltham, MA, USA) for equimolar sample pooling $(1.5 \mathrm{pM})$. Sequencing reads were generated using the $2 \times 150$ (300 cycles) for the base-read length chemistry of the Illumina MiniSeq platform.

\section{Shrimp gut microbial taxonomy and diversity analysis}

The software Shaman (http://shaman.c3bi.pasteur.fr/) and MicrobiomeAnalyst (https://www.microbiomeanalyst.ca/) were used to analyze the sequences from the Illumina MiSeq ( $2 \times 150$ pair-end). Sequence cleaning was performed with pair-end cleaner v1.0.3. The following operational taxonomic units (OTUs) construction process was carried out on the Shaman 
platform: read quality control, dereplication, removing singletons, removing of chimera sequences, and grouping. The annotation process (16S rDNA gene) was performed against the SILVA database with a confidence threshold of 0.8 (Shaman). The alpha indices (Shannon, Simpson, Chao 1, ACE) were estimated, and beta index analysis (PCoA) was performed to analyze the similarities/differences of the microbial communities between different treatments and a relative abundance analysis on the MicrobiomeAnalyst platform.

The metabolic potential of the shrimp gut bacterial community was analyzed with the multimodular web platform iVikodak (Nagpal et al. 2019). The Global Mapper module (independent contribution algorithm) was used to infer functional profiles and perform meaningful analyzes using the KEGG (metabolism) database for annotation. This module analyzes the metabolic pathways of microbial communities, estimates their relative abundance, quantifies the contribution of each taxon in a certain metabolic pathway, and identifies the main set of metabolic functions that define a particular environment (Ogata et al. 1999, Nagpal et al. 2016).

\section{Statistical analysis}

The analysis of alpha diversity (Shannon, Simpson, Chao 1, ACE) was carried out using ANOVA and a $t$ test (MicrobiomeAnalyst, $P<0.05$ ). For the beta index, the PCoA and ANOSIM test (Analysis of Group Similarities, MicrobiomeAnalyst, $P<0.05)$ were performed. To determine the differences in the relative abundance of the taxa, a one-way ANOVA, followed by a Tukey honest significant discriminant (HSD) test (Statistica Software, StatSoft, $P<0.05$ ), and the Wald test $(P<0.05)$ were performed. Wald test was based on model parameters defined by the DESeq2 package run in the Shaman platform.

\section{RESULTS}

The total reads obtained from the NGS sequencing for both inulin and control conditions were 651,865 reads. For control conditions (A) 343,346 reads, and inulin treatment (B) 308,519 reads were obtained, respectively. After the quality control reads, chimeric sequences, low-quality bases and sequences, and singletons were removed. Then, read clustering and taxonomic assignments at $97 \%$ identity with the Silva database were obtained. Among 331 OTUs, 150 OTUs belong to inulin treatment, and 181 OTUs for control conditions were identified in the shrimp intestine.

The microbial community of shrimp intestine consisted of 9 phyla, 13 classes, 30 orders, 37 families,
71 genera, and 17 species. The shrimp gut microbial community structure showed that Proteobacteria was the dominant phylum, followed by Bacteroidetes (Fig. 1). Vibrionaceae and Rhodobacteraceae were the most abundant families, whereas Vibrio and Ruegeria were the most abundant genera.

The relative abundance changes of the most relevant phyla, class, order, family, and genus were determined in the intestinal shrimp microbiome. The dominant phyla for both inulin treatment and control conditions were Proteobacteria, Bacteroidetes, and Patescibacteria. The phylum Bacteroidetes underwent a significant decrease $(P=0.006)$ in the relative abundance for inulin-treated shrimps $(5.16 \pm 3.89 \%)$ concerning the control group $(16.95 \pm 9.39 \%)$. The class Bacteroidia showed a significant decrease $(P=$ $0.011)$ for inulin-treated shrimps $(6.93 \pm 3.98 \%)$ concerning the control condition shrimps (16.68 \pm $8.73 \%)$. The order Flavobacteriales showed a significant decrease $(P=0.006)$ in inulin-treated shrimps $(4.73 \pm 3.71 \%)$ compared to the control group $(14.80 \pm 9.30 \%)$. The family Flavobacteriaceae underwent a significant decrease $(P=0.009)$ for inulintreated shrimps $(4.89 \pm 3.46 \%)$ in comparison to the control group $(14.65 \pm 9.25 \%)$. The genus Ruegeria underwent a significant decrease $(P=0.029)$ for inulin treated shrimps $(11.89 \pm 8.18 \%)$ with respect to control group $(22.22 \pm 10.39)$ (Table 1$)$.

The estimated alpha indices (Shannon, Simpson, ACE, and Chao1) in all taxa did not show significant differences between B and A. Alpha indices for richness and diversity were only shown at the genus level (Table 2).

The principal coordinate analysis (PCoA) based on the Bray-Curtis index was carried out to determine the differences/similarities in the shrimp gut microbial community. At the genus level, not significant differences were found in diversity $(P<0.07)$ between samples for B and A. The PCoA showed that most samples of the inulin treatment and control condition overlapping (Fig. 2). Most of the gut bacterial communities of shrimps were not separated from inulin treatment and the control condition, so a lack of individual variation. Variations within each group were not detected.

This study carried out a microbiota functional analysis profile for both control condition (Fig. 3a) and inulin treatment (Fig. 3b) and was supported by the KEGG database. For inulin treated samples, six functional categories were found, including metabolism (62\%), human disease (11\%), genetic information processing $(10 \%)$, organismal systems $(8 \%)$, cellular processes (7\%), and environmental information processing $(2 \%)$. For control condition samples, six 


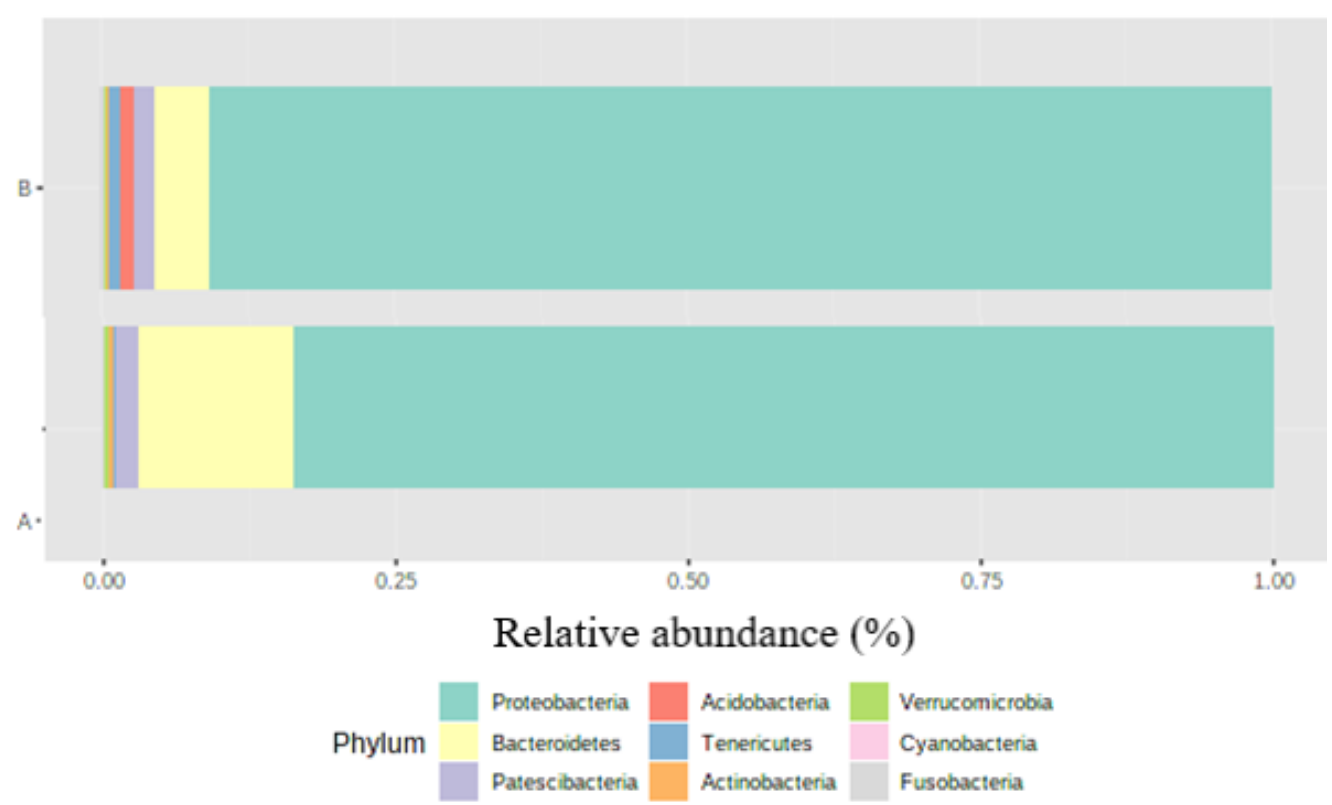

Figure 1. Bacterial relative abundance (\%) at phylum level in Penaeus vannamei intestines. A: Control condition, and B: inulin treatment. Only bacteria with a relative abundance $\geq 1 \%$ are reported (Shaman).

Table 1. Mean relative abundance (\%) of the most relevant phyla, classes, orders, families, and genera found in intestine samples of Penaeus vannamei fed diets without inulin (A: control condition) and with inulin (B: treatment). Data are mean \pm standard deviation. Statistical analysis: one-way ANOVA and Tukey HSD test. The same letter means no significant difference $(P<0.05)$.

\begin{tabular}{lccc}
\hline & A & B & $P$-value \\
\hline Phylum & & & \\
Proteobacteria & $80.63 \pm 12.94^{\mathrm{a}}$ & $88.44 \pm 6.84^{\mathrm{a}}$ & $>0.05$ \\
Bacteroidetes & $16.95 \pm 9.39^{\mathrm{a}}$ & $5.16 \pm 3.89^{\mathrm{b}}$ & 0.006 \\
Patescibacteria & $3.13 \pm 1.98^{\mathrm{a}}$ & $5.58 \pm 5.42^{\mathrm{a}}$ & $>0.05$ \\
Class & & & \\
Gammaproteobacteria & $40.09 \pm 27.09^{\mathrm{a}}$ & $58.27 \pm 19.98^{\mathrm{a}}$ & $>0.05$ \\
Bacteroidia & $16.68 \pm 8.73^{\mathrm{a}}$ & $6.93 \pm 3.98^{\mathrm{b}}$ & 0.011 \\
Alphaproteobacteria & $40.40 \pm 16.53^{\mathrm{a}}$ & $30.09 \pm 15.30^{\mathrm{a}}$ & $>0.05$ \\
Order & & & \\
Rhodobacterales & $39.03 \pm 16.61^{\mathrm{a}}$ & $26.30 \pm 16.51^{\mathrm{a}}$ & $>0.05$ \\
Vibrionales & $33.83 \pm 29.45^{\mathrm{a}}$ & $56.44 \pm 22.01^{\mathrm{a}}$ & $>0.05$ \\
Flavobacteriales & $14.80 \pm 9.30^{\mathrm{a}}$ & $4.73 \pm 3.71^{\mathrm{b}}$ & 0.006 \\
Family & & & \\
Rhodobacteraceae & $38.94 \pm 15.57^{\mathrm{a}}$ & $27.039 \pm 15.64^{\mathrm{a}}$ & $>0.05$ \\
Vibrionaceae & $33.83 \pm 29.45^{\mathrm{a}}$ & $52.35 \pm 23.96^{\mathrm{a}}$ & $>0.05$ \\
Flavobacteriaceae & $14.65 \pm 9.25^{\mathrm{a}}$ & $4.89 \pm 3.46^{\mathrm{b}}$ & 0.009 \\
Genus & & & \\
Vibrio & $32.22 \pm 29.41^{\mathrm{a}}$ & $49.74 \pm 26.69^{\mathrm{a}}$ & $>0.05$ \\
Ruegeria & $22.22 \pm 10.39^{\mathrm{a}}$ & $11.89 \pm 8.18^{\mathrm{b}}$ & 0.029 \\
Loktanella & $3.00 \pm 0.82^{\mathrm{a}}$ & $2.08 \pm 0.86^{\mathrm{a}}$ & $>0.05$ \\
Pseudoalteromonas & $3.38 \pm 3.34^{\mathrm{a}}$ & $3.16 \pm 2.49^{\mathrm{a}}$ & $>0.05$ \\
\hline
\end{tabular}

functional categories were found, including metabolism (64\%), human diseases (11\%), genetic information processing $(9 \%)$, organismal systems $(9 \%)$, cellular processes (6\%), and environmental information processing $(1 \%)$. No significant change was determined in the functions of the intestinal microbiota of the white shrimp Penaeus vannamei for inulin treatment compared to the control condition. The KEGG analysis 
Table 2. Alpha indices (genus level) in Penaeus vannamei intestines of control condition (A) and inulin treatment (B). Microbiome Analyst obtained indices. Data are mean \pm standard deviation.

\begin{tabular}{lcr}
\hline Indices & \multicolumn{1}{c}{$\mathrm{A}$} & \multicolumn{1}{c}{$\mathrm{B}$} \\
\hline Shannon & $2.21 \pm 0.83$ & $1.94 \pm 0.84$ \\
Simpson & $0.72 \pm 0.25$ & $0.65 \pm 0.25$ \\
Chao1 & $92.85 \pm 12.22$ & $88.63 \pm 7.47$ \\
ACE & $88.14 \pm 9.4$ & $91.91 \pm 8.40$ \\
\hline
\end{tabular}

showed that most of the metabolism sequences in the intestine of inulin treated group corresponded to carbohydrate metabolism (29\%) and amino acid metabolism (29\%), and a lesser extent to energy metabolism (13\%), lipid metabolism (14\%), and cofactors and vitamin metabolism (14\%) (Fig. 4). For the control group, the KEGG analysis showed that most of the metabolism sequences corresponded to carbohydrate metabolism $(30 \%)$ and amino acid metabolism (29\%), and a lesser extent to energy metabolism (13\%), lipid metabolism (13\%), and cofactors and vitamin metabolism (13\%) (Fig. 4). No significant change was observed in the metabolic functions of the shrimp intestinal microbiota under inulin treatment compared to those of the control condition (Fig. 4).
The top metabolic functions were identified in the intestinal microbiota of shrimp. For inulin treated group, the KEGG functional analysis showed that glycine, serine, and threonine metabolism represented the $15.9 \%$ of annotations, glyoxylate and dicarboxylate metabolism $12 \%$, purine metabolism $24.1 \%$, pyrimidine metabolism $17.7 \%$, pyruvate metabolism $14.4 \%$, and quorum sensing-related metabolism $14.96 \%$ (Fig. 5). For control condition samples, the KEGG functional analysis showed glycine, serine, and threonine metabolism (17.2\%), glyoxylate and dicarboxylate metabolism (14.2\%), purine metabolism (23.1\%), pyrimidine metabolism (17.5\%), pyruvate metabolism (14.6\%), and quorum sensing-related metabolism (13.0\%) (Fig. 5). No significant change was determined for the microbial metabolic functions between shrimp treated inulin and the control group (Fig. 5).

The functional interaction network analysis for the shrimp intestine inulin treatment showed that the network core was formed by Vibrio, Mesonia, Pseudoruegeria, Aeromonas, and Winogradskyella. For control shrimp, the network core was formed by Ruegeria, Vibrio, Pseudoruegeria, Tenacibaculum, Actibacterium, and Winogradskyella. The shrimp treated with inulin showed fewer interactions ([positive

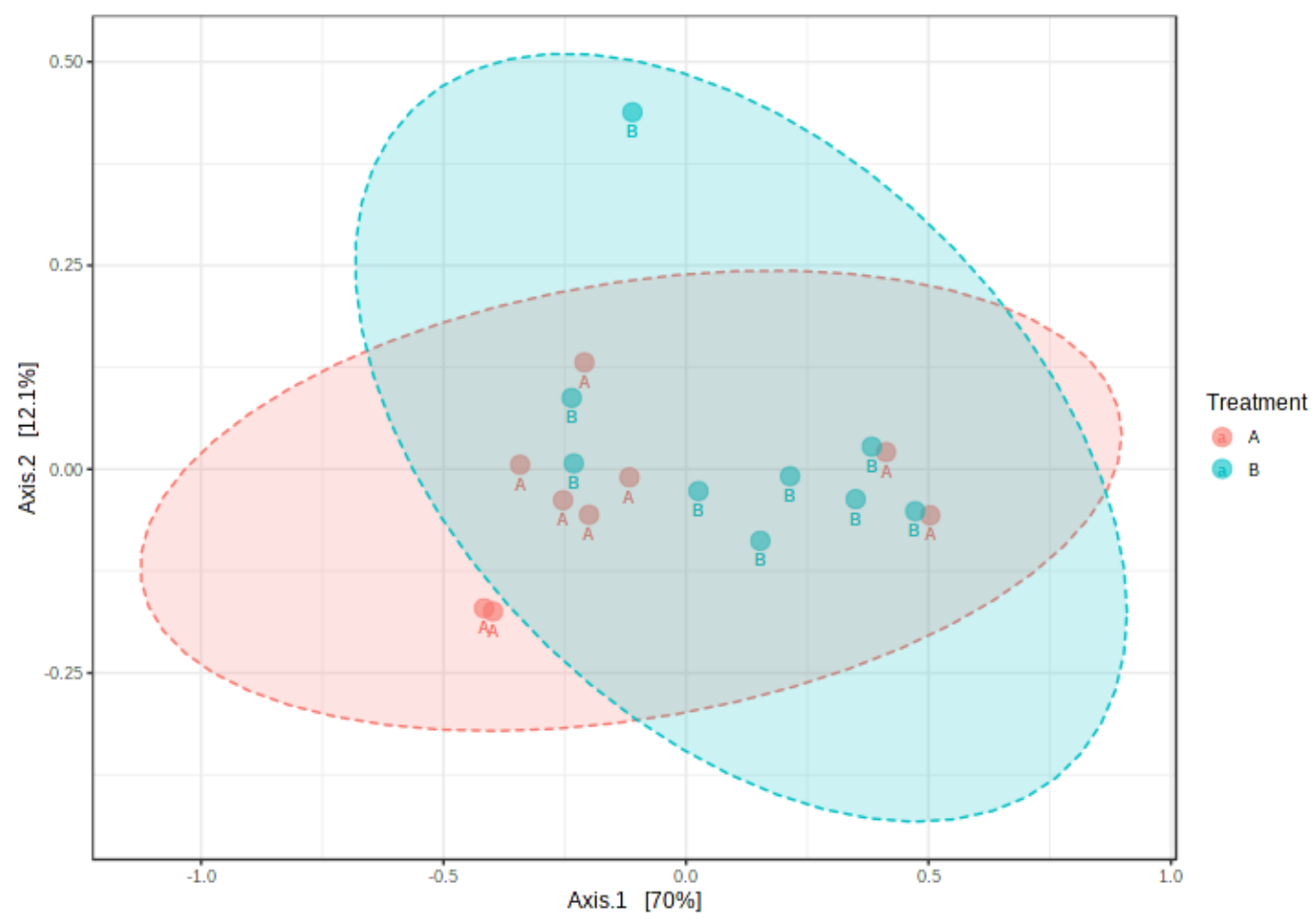

Figure 2. Principal coordinate analysis of bacterial (genus) populations associated with Penaeus vannamei intestines of control condition (A) and inulin treatment (B) (ANOSIM test, Bray-Curtis dissimilarity distance matrix, R: 0.119, $P<0.07$ ). 


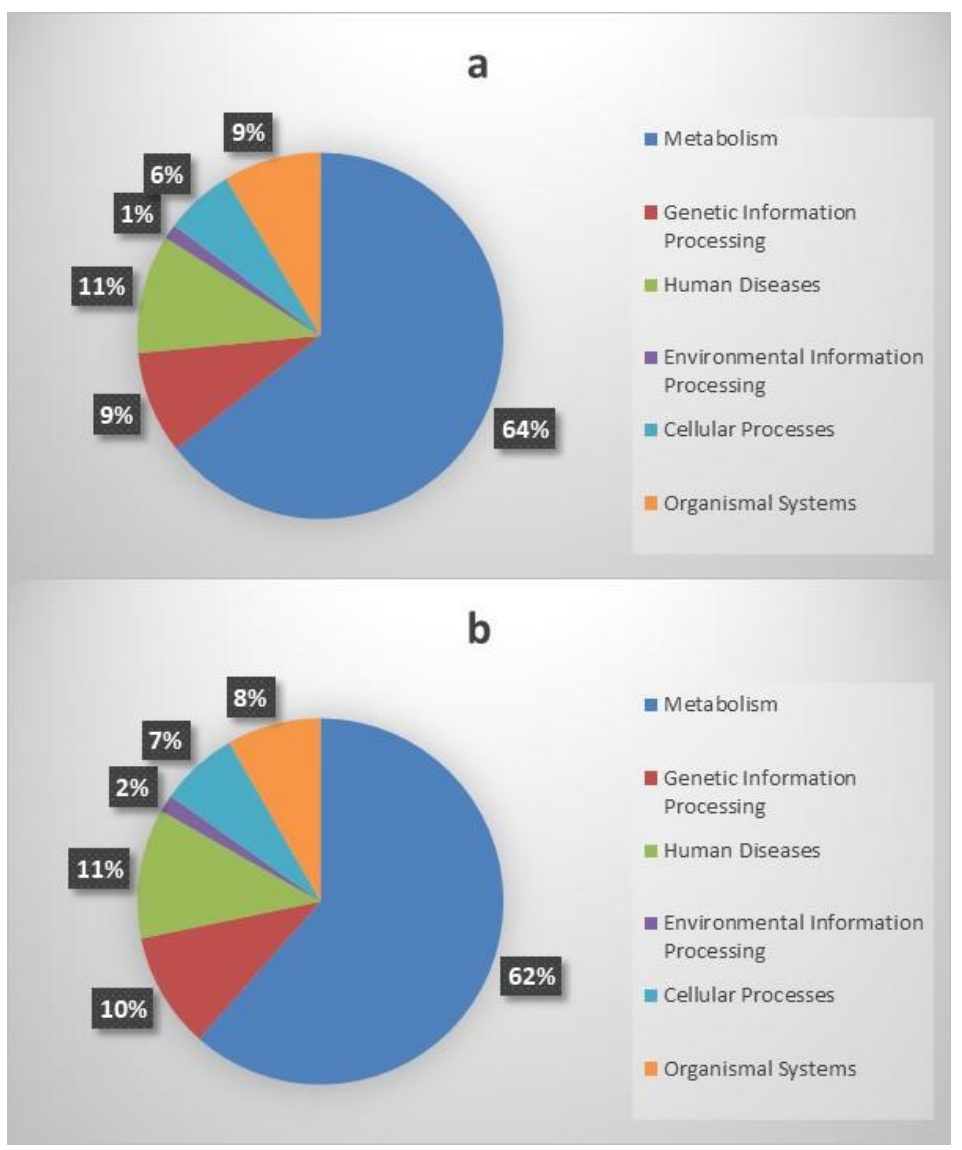

Figure 3. The top KEGG functional categories found in shrimp intestine samples. a) Control condition, b) inulin treatment.

= blue line] and negative [red line]) than that of the control shrimp. It is worth noting that negative interactions against Vibrio were established by the Streptomyces, Roseobacter, and Ruegeria genera (Figs. 6-7).

\section{DISCUSSION}

Nowadays, there is a lack of information about the role of the intestinal microbiota of aquatic invertebrates (Gao et al. 2019). Therefore, in this work, an analysis of the microbial community profile and function of the intestinal microbiota was performed in the white shrimp fed the prebiotic inulin. At several taxonomic levels, the sequence analysis showed that the most abundant phyla were Protobacteria and Bacteroidetes, both in the control condition and in the inulin treatment. However, the predominant phylum was Proteobacteria with a relative abundance of approximately $80 \%$, as reported in previous works carried out in shrimp cultured in laboratory-controlled conditions (Qiao et al. 2016, Sha et al. 2016, Zhang et al. 2016, Zheng et al. 2016, Vargas-Albores et al. 2017) and commercial farms (Gainza et al. 2017, Gao et al. 2019). At the class level, Gammaproteobacteria and Alphaproteobacteria were the predominant classes. Zheng et al. (2016) established the high relative abundance of Meridianimaribacter, Vibrio, Ruegeria, Pseudoalteromonas, and Tenacibaculum genera for healthy gut shrimps. In our study, Vibrio, Ruegeria, Photobacterium, Pseudoalteromonas, Tenacibaculum, and Maribacter genera were the most abundant as previously reported (Zheng et al. 2016, Suo et al. 2017, Amoah et al. 2019).

In this work, the most abundant genus was Vibrio, followed by Ruegeria, in which a significant decrease was determined for inulin-fed shrimp concerning control condition. Although most Vibrio are nonpathogenic (Noriega-Orozco et al. 2007), several Vibrio species cause diseases in farmed shrimp, such as $V$. parahaemolyticus $\mathrm{N} 1 \mathrm{~A}$ and $\mathrm{N} 7 \mathrm{~A}$ and $V$. harveyi N2A, N8A, N10A in Penaeus monodon (Stalin \& Srinivasan 2016, 2017), V. parahaemolyticus ATCC 17802 (Lomelí-Ortega \& Martínez-Díaz 2014), V. parahaemolyticus 13-028/A3- AHPND (Jun et al. 2018), Vibrio campbellii and Vibrio owensii (Dong et al. 2017a,b) in P. vannamei. Conversely, Ruegeria spp. showed antibacterial activity against $V$. anguillarum in a Danish turbot (Scophthalmus maximus) larval culture 

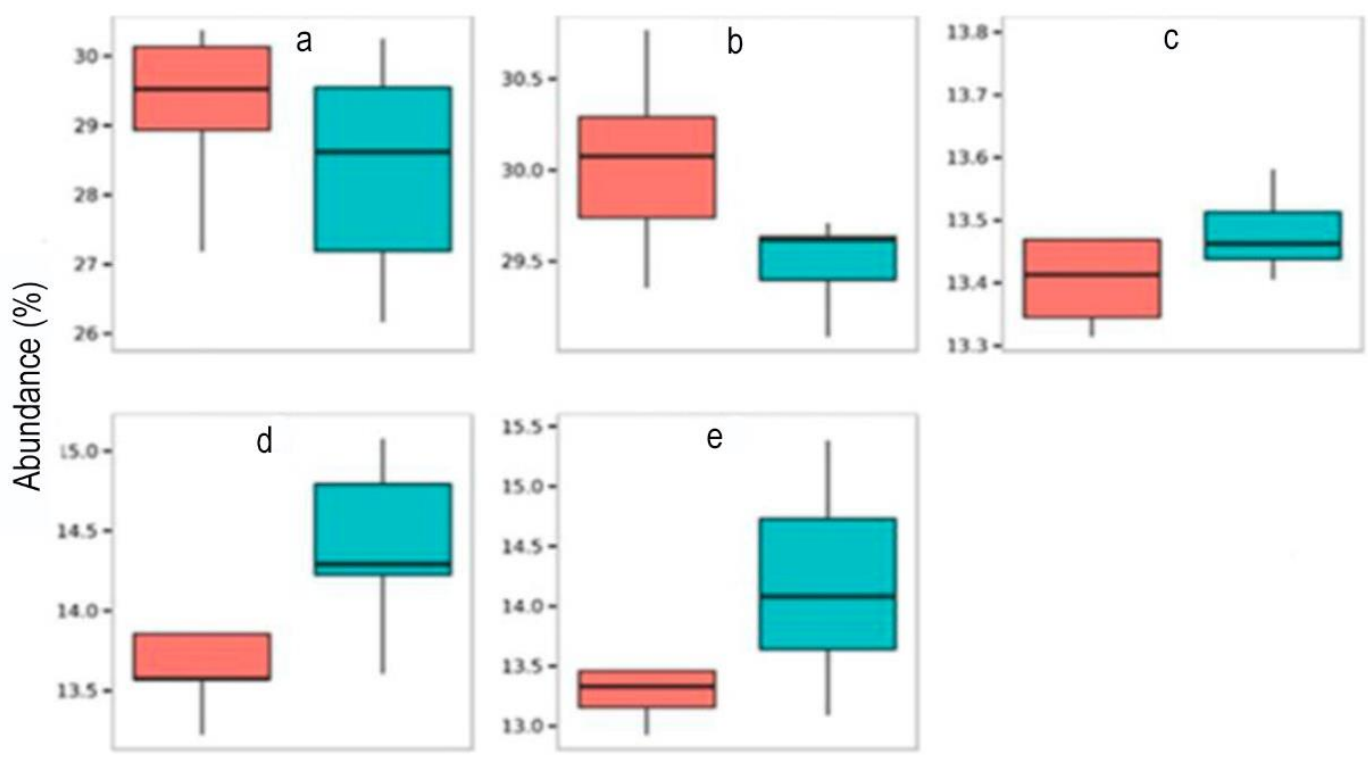

\section{Treatments 由 Control 官 Inulin}

Figure 4. Metabolism functional categories found in shrimp intestine samples for control condition and inulin treatment (iVikodak). a) Aminoacid metabolism, b) carbohydrate metabolism, c) energy metabolism, d) lipid metabolism, e) metabolism of cofactors and vitamins.
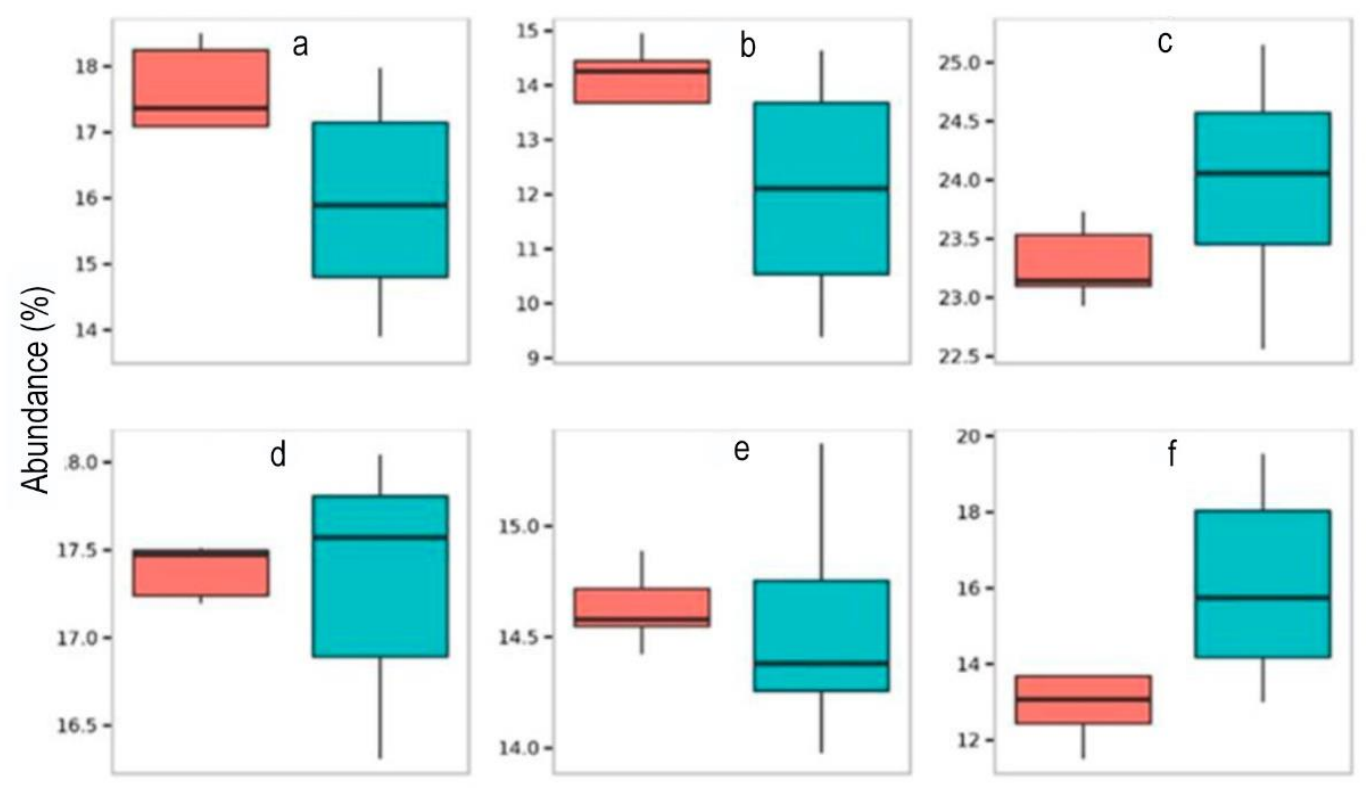

Treatments 官 Control 官 Inulin.

Figure 5. Top metabolic functions found in shrimp intestine samples for control condition and inulin treatment (iVikodak). a) Glycine, serine and threonine metabolism, b) glyoxylate and decarboxylate metabolism, c) purine metabolism, d) pyrimidine metabolism, e) pyruvate metabolism, f) quorum sensing.

(Porsby et al. 2008). Furthermore, Ruegeria has been shown to have triesterase activity, contributing to host digestive processes (Yamaguchi et al. 2016). It suggests that the presence of Vibrio spp. and Ruegeria spp. may plays a competitive role in aquatic animals. 


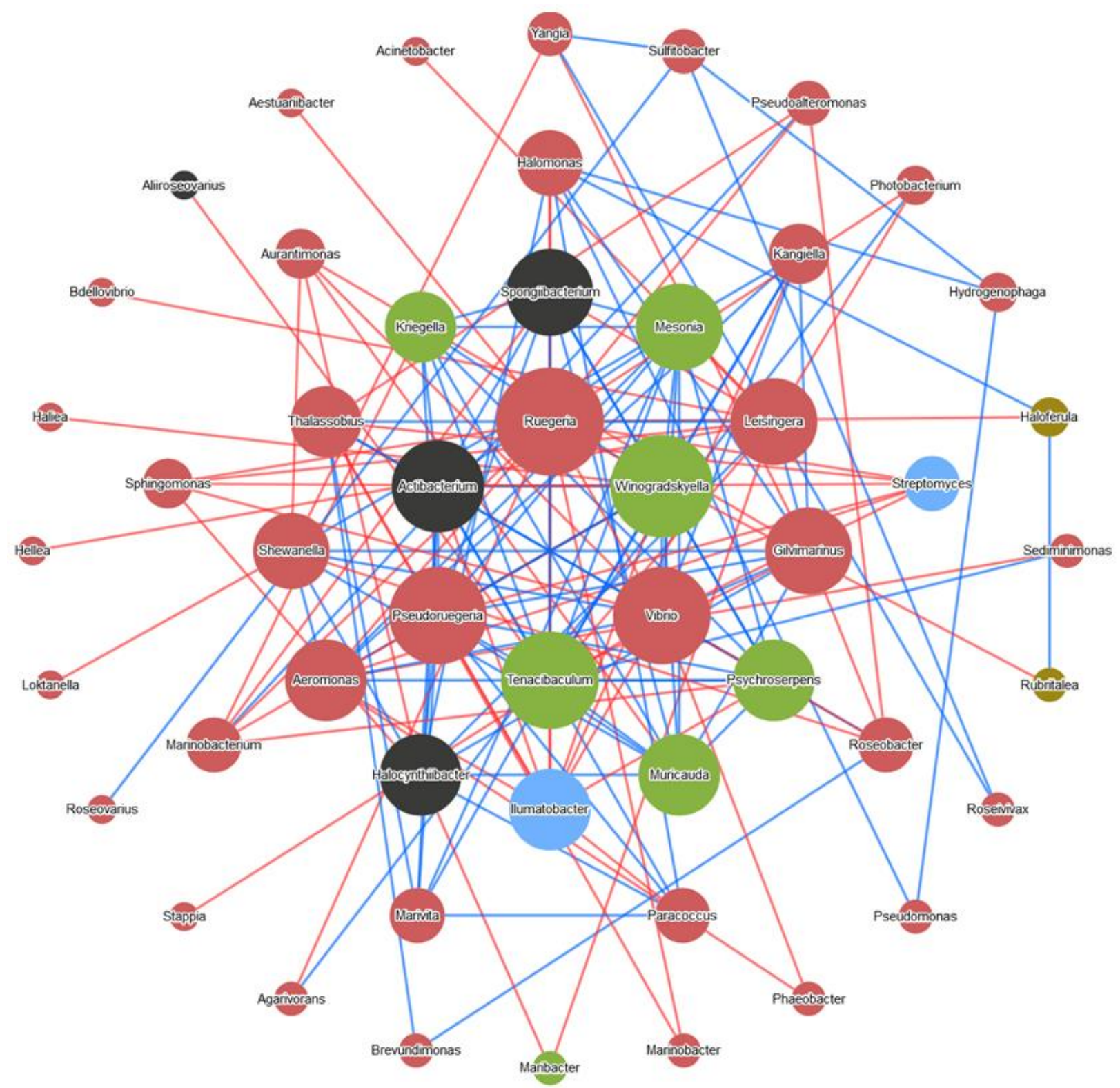

Figure 6. Functional interaction networks at the genus level found in intestine samples of Penaeus vannamei fed with control condition diet. Red nodes: Proteobacteria, green nodes: Bacteroidetes, blue nodes: Actinobacteria, olive green nodes: Verrucomicrobia, black nodes: others (iVikodak). Large nodules indicate a high degree of interaction. The blue lines indicate positive interactions (cooperative interaction), and the red lines indicate negative interactions (non-cooperative interaction). The genera with the largest nodes also indicate their importance in the shrimp intestine microbial community.

Shannon and Simpson are diversity indices commonly used in the measurement of bacterial diversity based on OTU. Bacterial diversity refers to the number of species and their numerical composition of a bacterial community in a certain niche (Schloss \& Handelsman 2005, 2006, Schloss et al. 2009, Kim et al. 2017). On the other hand, the ACE and Chao1 indices estimate the total species richness of a bacterial community in a sample (Hughes et al. 2001, Chao et al. 2006). In this study, the estimated alpha indices did not show significant differences in all taxa between inulin treatment and control conditions; therefore, inulin did not increase microbial diversity. It is known that high microbial diversity is considered to provide functional redundancy in an ecosystem, making it more stable and resistant to environmental stress (Turnbaugh et al. 2008, Le Chatelier et al. 2013). However, Fan et al. (2019) carried out a study in the intestine of $P$. vannamei and determined that the increase in shrimp growth was due to the influence of the microbiota on 


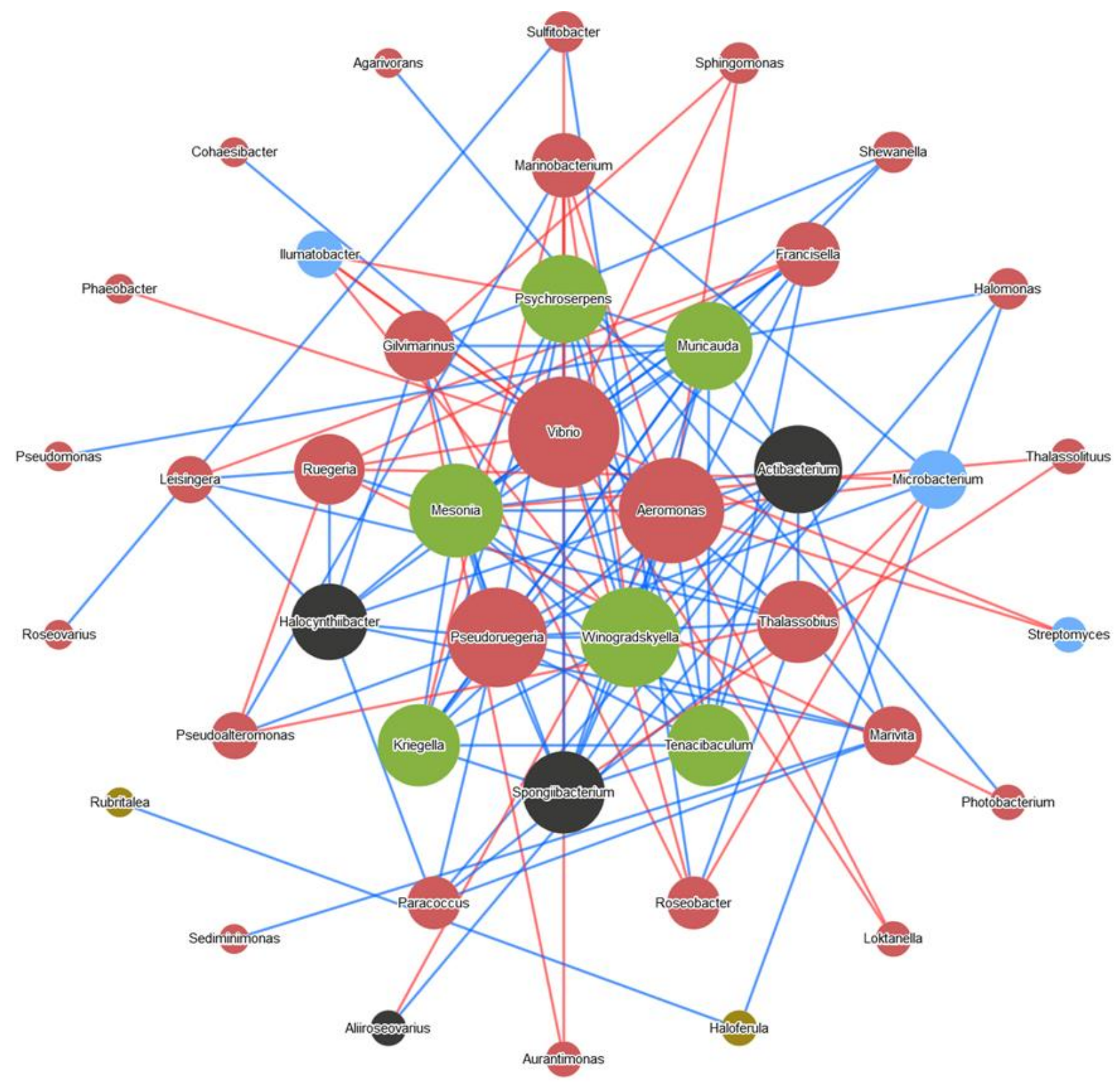

Figure 7. Functional interaction networks at the genus level found in intestine samples of Penaeus vannamei fed with inulin diet. Red nodes: Proteobacteria, green nodes: Bacteroidetes, blue nodes: Actinobacteria, olive green nodes: Verrucomicrobia, black nodes: others (iVikodak). Large nodules indicate a high degree of interaction. The blue lines indicate positive interactions (cooperative interaction), and the red lines indicate negative interactions (non-cooperative interaction). The genera with the largest nodes also indicate their importance in the shrimp intestine microbial community.

nutrient absorption rather than microbial diversity. In addition, Beta diversity analyzes the difference in the composition of the bacterial community for different environments (Tuomisto 2010). In this work, most of the bacterial communities in the intestines of shrimps from the control condition and inulin treatment were not separated from one another, suggesting the lack of individual variation. Therefore, there were no significant differences between the intestinal bacterial communities. Similarly, Landsman et al. (2019) found that the intestines of indoor-cultured shrimps showed homogenous bacterial communities. It is important to mention that the resolution of the analysis only reaches the genus, and we do not know if there are changes in diversity and abundance at the species level and their possible impact on the host.

Bacteria possess a plethora of enzymes for hydrolysis of a wide variety of fibers, including 
polysaccharide lyases, glycoside hydrolases, glycosyltransferases, and carbohydrate esterases (Cummings \& Macfarlane 1991, El Kaoutari et al. 2013). In this sense, many of the bacteria genera found, such as Ruegeria, Kriegella, Aurantimonas, and Mesonia, probably do not contain carbohydrase enzymes necessary to break down the bonds of inulin, such as Fusibacter, Pseudomonas, and Thalassolitus must-have. As observed, the inulin feeding did not enhance the variety of the shrimp intestinal microbial community, but the opposite, giving rise to the increase of Proteobacteria, Vibrionaceae, and Vibrio, which are highly pathogenic strains for shrimp. In humans, a diet with a variety of fibers (e.g. pectins, fructans, cellulose, hemicelluloses, pectins, gums) give rise to enhance the intestinal microbial diversity (Cummings \& Macfarlane 1991, El Kaoutari et al. 2013); this suggests that it is necessary to test diets with different prebiotics, which should be suitable for invertebrates to promote intestinal microbial diversity.

The microbial community in shrimp intestines had a high content of genes related to metabolism, human diseases, processing of genetic information, and organismal systems. However, metabolism was highly represented in this study (62-64\%) which may be related to energy consumption to satisfy the physiological activities of the host (Wang et al. 2015). Among the KEEG metabolism subcategories, most functional categories corresponded to carbohydrate and amino acid and, to a lesser extent, energy, lipid, and cofactor, and vitamin metabolism. According to Wang et al. (2015), the presence of these functional subcategories in humans showed that the metabolic potential of bacteria in the intestine is highly diverse and versatile. They are well adapted for the degradation of carbohydrates and amino acids. Similar results have been demonstrated in turbot (S. maximus) by Xing et al. (2013).

According to Nagpal et al. (2016), the interaction network analysis showed the functional interaction between microorganisms and the synchronization (positive interactions in the blue line)/antagonism (negative interactions in the red line) that occurs as a result of the specific physiological conditions. The largest nodes indicate that the microorganisms have a key functional role in the community. Also, the networks generated by two communities allow identifying changes in members with a critical role in the community and the transition in the general interactions between the resident microorganisms (Nagpal et al. 2016). In this study, the control condition network showed core genera (Ruegeria, Vibrio, Pseudoruegeria, Tenacibaculum, Actibacterium, and Winogradskyella) with large nodes and the highest number of interactions. In the inulin treatment, large nodes (genera) were observed with fewer interactions composed of Vibrio, Mesonia, Pseudoruegeria, Aeromonas, and Winogradskyella. Similarly, Li et al. (2016) mention that highly connected nodes tend to be functionally similar or to interact closely to establish a tight unit for a specific biological function.

In conclusion, the shrimp intestinal microbiota predominated in all samples belongs to the phyla Proteobacteria and Bacteroidetes, and Vibrio and Ruegeria, two genera with pathogenic and probiotic species, respectively. This work provides evidence that the dietary inulin did not change diversity in the intestinal shrimp microbiota, but the bacterial metabolism plays an important role, mainly in the breakdown of carbohydrates and amino acids, with complex interactions. Finally, based on negative bacterial interactions with Vibrio, we can recommend Streptomyces, Roseobacter and Ruegeria as potential probiotics for shrimp.

\section{ACKNOWLEDGMENTS}

This work was supported by Secretaría de Investigación y Posgrado del Instituto Politécnico Nacional (SIP-IPN, grant 20181360) for financial support.

\section{REFERENCES}

Abdulrahman, N.M. \& Ahmed, V.M. 2015. Comparative effect of probiotic (Saccharomyces cerevisiae), prebiotic (fructooligosaccharides FOS) and their combination on some differential white blood cells in young common carp (Cyprinus carpio L.). Asian Journal of Science and Technology, 6: 1136-1140.

Aich, N., Ahmed, N. \& Paul, A. 2018. Issues of antibiotic resistance in aquaculture industry and its way forward. International Journal of Current Microbiology and Applied Science, 7: 26-41. doi: 10.20546/ijcmas.2018. 708.004

Amoah, K., Huang, Q.C., Tan, B.P., Zhang. S., Chi, S.Y., Yang, Q.H., et al. 2019. Dietary supplementation of probiotic Bacillus coagulans ATCC 7050 improves the growth performance, intestinal morphology, microflora, immune response, and disease confrontation of Pacific white shrimp, Litopenaeus vannamei. Fish Shellfish of Immunology, 87: 796-808. doi: 10.1016/j.fsi.2019.02.029

Azimirad, M., Meshkini, S., Ahmadifard, N. \& Hoseinifar, S.H. 2016. The effects of feeding with synbiotic (Pediococcus acidilactici and fructooligosaccharide) enriched adult Artemia on skin mucus immune responses, stress resistance, intestinal microbiota, and performance of angelfish (Pterophyllum 
scalare). Fish Shellfish of Immunology, 54: 516-522. doi: 10.1016/j.fsi.2016.05.001

Chao, A., Chazdon, R.L., Colwell, R.K. \& Shen, T.J. 2006. Abundance-based similarity indices and their estimation when there are unseen species in samples. Biometrics, 62: 361-371. doi: 10.1111/j.1541-0420. 2005.00489.x

Cummings, J.H. \& Macfarlane, G.T. 1991. The control and consequences of bacterial fermentation in the human colon. Journal of Applied Bacteriology, 70: 443-459. doi: 10.1111/j.1365-2672.1991.tb02739.x

Dong, X., Wang, H., Xie, G., Zou, P., Guo, C., Liang, Y. \& Huang, J. 2017a. An isolate of Vibrio campbellii carrying the pirVP gene causes acute hepatopancreatic necrosis disease. Emerging Microbes \& Infections, 6: 1-3. doi: 10.1038/emi.2016.131

Dong, X., Bi, D., Wang, H., Zou, P., Xie, G. \& Wan, X., Yang Q., et al. 2017b. pir- $\mathrm{AB}^{\text {vp }}$-bearing Vibrio parahaemolyticus and Vibrio campbellii pathogens isolated from the same AHPND-affected pond possess highly similar pathogenic plasmids. Frontiers in Microbiology, 8: 1859. doi: 10.3389/fmicb.2017.018 59

El Kaoutari, A., Armougom, F., Gordon, J.I., Raoult, D. \& Henrissat, B. 2013. The abundance and variety of carbohydrate-active enzymes in the human gut microbiota. Nature Reviews Microbiology, 11: 497504. doi: 10.1038/nrmicro3050

Fan, J., Chen, L., Mai, G., Zhang, H., Yang, J., Deng, D. \& Ma, Y. 2019. Dynamics of the gut microbiota in developmental stages of Litopenaeus vannamei reveal its association with body weight. Scientific Reports, 9: 734. doi: 10.1038/s41598-018-37042-3

Gao, S., Luqing, P., Huang, F., Mengsi, S., Changcheng, T. \& Mengyu, Z. 2019. Metagenomic insights into the structure and function of intestinal microbiota of the farmed Pacific white shrimp (Litopenaeus vannamei). Aquaculture, 499: 109-118. doi: 10.1016/j.aquaculture.2018.09.026

Gainza, O., Ramírez, C., Ramos, A.S. \& Romero, J. 2017. Intestinal microbiota of white shrimp Penaeus vannamei under intensive cultivation conditions in Ecuador. Microbial Ecology, 75: 562-568.

Gibson, G.R. 2004. Fibre and effects on probiotics (the prebiotic concept). Clinical Nutrition Supplements, 1: 25-31. doi: 10.1016/j.clnu.2004.09.005

Hoseinifar, S.H., Zare, P. \& Kolangi, M.H. 2015. The effects of different routes of inulin administration on gut microbiota and survival rate of Indian white shrimp post-larvae (Fenneropenaeus indicus). Veterinary Research Forum, 6: 331-335.

Hughes, J.B., Hellmann, J.J., Ricketts, T.H. \& Bohannan, B.J. 2001. Counting the uncountable: statistical approaches to estimating microbial diversity. Applied and Environmental Microbiology, 67: 4399-4406. doi: 10.1128/AEM.67.10.4399-4406.2001

Jun, J.W., Han, J.E., Giri, S.S., Tang, K.F.J., Zhou, X., et al. 2018. Phage application for the protection from acute hepatopancreatic necrosis disease (AHPND) in Penaeus vannamei. Indian Journal of Microbiology, 58: 114-117. doi: 10.1007/s12088-017-0694-9

Kim, B.R., Jiwon, S., Guevarra, R.B., Lee, J.H., Kim, D.W., Seol, K.H., et al. 2017. Deciphering diversity indices for a better understanding of microbial communities. Journal of Microbiology Biotechnology, 27: 2089-2093. doi: 10.4014/jmb.1709.09027

Landsman, A., St-Pierre, B., Rosales-Leija, M., Brown, M. \& Gibbons, W. 2019. Impact of aquaculture practices on intestinal bacterial profiles of Pacific whiteleg shrimp Litopenaeus vannamei. Microorganisms, 7: 93. doi: 10.3390/microorganisms7040093

Le Chatelier, E., Nielsen T., Qin J., Prifti E., Hildebrand F., Falony, G., et al. 2013. Richness of human gut microbiome correlates with metabolic markers. Nature, 500: 541-546. doi: 10.1038/ nature 12506

Li, L., Zicheng, W., Peng, H., Shining, M., Jie, D. \& Rui, J. 2016. Construction and analysis of functional networks in the gut microbiome of type 2 diabetes patients. Genomics Proteomics Bioinformatics, 14: 314-324. doi: 10.1016/j.gpb.2016.02.005

Li, E., Chang, X., Xiaodan, W., Shifeng, W., Qun, Z., Meiling, Z.J., et al. 2018. Gut microbiota and its modulation for healthy farming of Pacific white shrimp Litopenaeus vannamei. Reviews in Fisheries Science \& Aquaculture, 26: 381-399. doi: 10.1080/233 08249.2018.1440530

Lomelí-Ortega, C.O. \& Martínez-Díaz, S.F. 2014. Phage therapy against Vibrio parahaemolyticus infection in the whiteleg shrimp (Litopenaeus vannamei) larvae. Aquaculture, 434: 208-211. doi: 10.1016/j.aquaculture.2014.08.018

Luna-González, A., Almaraz-Salas, J.C., FierroCoronado, J.A., Flores-Miranda, M.C., GonzálezOcampo, H.A. \& Peraza-Gómez, V. 2012. The prebiotic inulin increases the phenoloxidase activity and reduces the prevalence of WSSV in whiteleg shrimp (Litopenaeus vannamei) cultured under laboratory conditions. Aquaculture, 362-363: 28-32. doi: 10.1016/j.aquaculture.2012.07.022

Makki, K., Deehan, E.C., Walter, J. \& Backhed, F. 2018. The impact of dietary fiber on gut microbiota in host health and disease. Cell Host \& Microbe, 23: 705-715.

Nagpal, S., Haque, M.M. \& Mande, S.S. 2016. Vikodak a modular framework for inferring functional potential of microbial communities from $16 \mathrm{~S}$ metagenomic datasets. Plos One, 11: e0148347. doi: 10.1371/journal.pone. 0148347 
Nagpal, S., Haque, M.M., Singh, R. \& Mande, S.S. 2019. iVikodak- a platform and standard workflow for inferring, analyzing, comparing, and visualizing the functional potential of microbial communities. Frontiers in Microbiology, 9: 3336. doi: 10.3389/ fmicb.2018.03336

Noriega-Orozco, L., Acedo-Félix, E., Higuera-Ciapara, I., Jiménez-Flores, R. \& Cano, R. 2007. Pathogenic and non pathogenic Vibrio species in aquaculture shrimp ponds. Revista Latinoamericana de Microbiología, 49: 60-67.

Ogata, H., Goto, S., Sato, K., Fujibuchi, W., Bono, H. \& Kanehisa, M. 1999. KEGG: Kyoto encyclopedia of genes and genomes. Nucleic Acids Research, 27: 2934. doi: 10.1093/nar/27.1.29

Porsby, C.H., Nielsen, K.F. \& Gram, L. 2008. Phaeobacter and Ruegeria species of the Roseobacter clade colonize separate niches in a Danish turbot (Scophthalmus maximus)-rearing farm and antagonize Vibrio anguillarum under different growth conditions. Applied Environmental Microbiology, 74: 7356-7364. doi: 10.1128/AEM.01738-08

Qiao, F., Liu, Y.K., Sun, Y.H., Wang, X.D., Chen, K., Li, T.Y. \& Zhang, M.L. 2016. Influence of different dietary carbohydrate sources on the growth and intestinal microbiota of Litopenaeus vannamei at low salinity. Aquaculture Nutrition, 23: 444-452. doi: 10.1111/anu. 12412

Ring $\varnothing$, E., Olsen, R.E., Gifstad, T.Ø., Dalmo, R.A., Amlund, H., Hemre, G.I. \& Bakke, A.M. 2010. Prebiotics in aquaculture: a review. Aquaculture Nutrition, 16: 117-136. doi: 10.1111/j.1365-2095.2009.00 731.x

Roberfroid, M. \& Slavin, J. 2000. Nondigestible oligosaccharides. Critical Reviews in Food Science and Nutrition, 40: 461-480.

Schloss, P.D. \& Handelsman, J. 2005. Introducing DOTUR, a. computer program for defining operational taxonomic units and estimating species richness. Applied Environmental Microbiology, 71: 1501-1506. doi: 10.1128/AEM.71.3.1501-1506.2005

Schloss, P.D. \& Handelsman, J. 2006. Introducing SONS, a tool for operational taxonomic unit-based comparisons of microbial community memberships and structures. Applied Environmental Microbiology, 72: 6773-6779. doi: 10.1128/AEM.00474-06

Schloss, P.D., Westcott, S.L., Ryabin, T., Hall, J.R., Hartmann, M. \& Hollister, E.B. 2009. Introducing mothur: open-source, platform-independent, community- supported software for describing and comparing microbial communities. Applied Environmental Microbiology, 75: 7537-7541. doi: 10.1128/AEM.015 41-09

Sha, Y., Mei, L., Wang, B., Jiang, K., Qi, C. \& Wang, L. 2016. Bacterial population in intestines of Litopenaeus vannamei fed different probiotics or probiotic supernatant. Journal Microbiology Biotechnology, 26: 1736-1745. doi: 10.4014/jmb.1603.03078

Stalin, N. \& Srinivasan, P. 2016. Characterization of Vibrio parahaemolyticus and its specific phage from shrimp pond in Palk Strait, South East coast of India. Biologicals, 1045-1056: 526-533. doi: 10.1016/j.biologicals.2016.08.003

Stalin, N. \& Srinivasan, P. 2017. Efficacy of potential phage cocktails against Vibrio harveyi and closely related Vibrio species isolated from shrimp aquaculture environment in the southeast coast of India. Veterinary Microbiology, 207: 83-96. doi: 10.1016/j.vetmic.2017.06.006

Suo, Y., Li, E., Li, T., Jia, Y., Qin, J.G., Gu, Z. \& Chen, L. 2017. Response of gut health and microbiota to sulfide exposure in Pacific white shrimp Litopenaeus vannamei. Fish and Shellfish Immunology, 63: 87-96. doi: 10.1016/j.fsi.2017.02.008

Teitelbaum, J.E. \& Walker, W.A. 2002. Nutritional impact of pre -and probiotics as protective gastrointestinal organisms. Annual Review of Nutrition, 22: 107-138. doi: 10.1146/annurev.nutr.22.110901.1454 12

Tuomisto, H. 2010. A diversity of beta diversities: straightening up a concept gone awry. Part 1. Defining beta diversity as a function of alpha and gamma diversity. Ecography, 33: 2-22. doi: 10.1111/j.16000587.2009.05880.x

Turnbaugh, P.J., Hamady, M., Yatsunenko, T., Cantarel, B.L., Duncan, A., Ley, R.E., et al. 2008. A core gut microbiome in obese and lean twins. Nature, 457: 480484. doi: 10.1038 /nature 07540

Vargas-Albores, F., Porchas-Cornejo, M.A., MartínezPorchas, M., Villalpando-Canchola, E., GollasGalván, T. \& Martínez-Córdova, L.R. 2017. Bacterial biota of shrimp intestine is significantly modified by the use of a probiotic mixture: a high throughput sequencing approach. Helgoland Marine Research, 71: 5. doi: 10.1186/s10152-017-0485-Z

Vulevic, J., Rastall, R.A. \& Gibson, G.R. 2004. Developing a quantitative approach for determining the in vitro prebiotic potential of dietary oligosaccharides. FEMS Microbiology Letters, 236: 153159. doi: 10.1016/j.femsle.2004.05.036

Wang, W.L., Xu, S.Y., Ren, Z.G., Tao, L., Jiang, J.W. \& Zheng, S.S. 2015. Application of metagenomics in the human gut microbiome. World Journal of Gastroenterology, 21: 803-814. doi: 10.3748/wjg.v21.i3.803

Xing, M., Hou, Z., Yuan, J., Liu, Y., Qu, Y. \& Liu, B. 2013. Taxonomic and functional metagenomic profiling of gastrointestinal tract microbiome of the farmed adult turbot (Scophthalmus maximus). FEMS Microbiology Ecology, 86: 432-43. doi: 10.1111/ 1574-6941.12174 
Yamaguchi, H., Arisaka, H., Seki, M., Adachi, M., Kimura, K. \& Tomaru, Y. 2016. Phosphotriesterase activity in marine bacteria of the genera Phaeobacter, Ruegeria, and Thalassospira. International Biodeterioration \& Biodegradration, 115: 186-191. doi: 10.1016/j.ibiod.2016.08.019

Zhang, M., Sun, Y., Liu, Y., Qiao, F., Chen, L., Liu, W.T. \& Li, E. 2016. Response of gut microbiota to salinity change in two euryhaline aquatic animals with reverse salinity preference. Aquaculture, 454: 72-80. doi: 10.1016/j.aquaculture.2015.12.014

Received: 22 October 2020; Accepted: 5 February 2021
Zheng, Y., Yu, M., Liu, Y., Su, Y., Xu, T., Yu, M. \& Zhang, X.H. 2016. Comparison of cultivable bacterial communities associated with Pacific white shrimp (Litopenaeus vannamei) larvae at different health statuses and growth stages. Aquaculture, 451: 163169. doi: 10.1016/j.aquaculture.2015.09.020 